\title{
The Best Constant of $L^{p}$ Sobolev Inequality Including $j$-th Derivative Corresponding to Periodic and Neumann Boundary Value Problem for $(-1)^{M}(d / d x)^{2 M}$
}

\author{
Hiroyuki YAMAGISHI and Kohtaro WATANABE \\ Tokyo Metropolitan College of Industrial Technology and National Defense Academy \\ (Communicated by A. Tachikawa)
}

Abstract. In this paper, we study the best constant of $L^{p}$ Sobolev inequality including $j$-th derivative:

$$
\sup _{0 \leq y \leq 1}\left|u^{(j)}(y)\right| \leq C\left(\int_{0}^{1}\left|u^{(M)}(x)\right|^{p} d x\right)^{1 / p},
$$

where $u$ is an element of Sobolev space with periodic or Neumann boundary condition. The best constant can be expressed by $L^{q}$ norm of Bernoulli polynomial. In [1,4], the best constant of the above inequality was obtained for the case of $1<p<\infty$ and $j=0$. This paper extends the results of $[1,4]$ to $j=1,2,3, \ldots, M-1$.

\section{Introduction}

Throughout this paper, we assume that $M=1,2,3, \ldots, p, q>1$ and $1 / p+1 / q=1$. We introduce the following notation for $L^{p}$ norm

$$
\|u\|_{p}=\left(\int_{0}^{1}|u(x)|^{p} d x\right)^{1 / p}
$$

and the series of Sobolev spaces

$$
W(\mathrm{X}, M, p)=\left\{u \mid u^{(M)} \in L^{p}(0,1), \quad u \text { satisfies } A(\mathrm{X})\right\}
$$

where the condition $A(\mathrm{X})$ assumes

$$
\begin{array}{lll}
A(\mathrm{P}): u^{(i)}(1)-u^{(i)}(0)=0 & (0 \leq i \leq M-1), & \int_{0}^{1} u(x) d x=0 \\
A(\mathrm{AP}): u^{(i)}(1)+u^{(i)}(0)=0 & (0 \leq i \leq M-1),
\end{array}
$$

Received August 27, 2013; revised November 12, 2013

2010 Mathematics Subject Classification: 34B27 (Primary), 46E35 (Secondary)

Key words and phrases: $L^{p}$ Sobolev inequality, Best constant, Green function, Reproducing kernel, Bernoulli polynomial, Hölder inequality

This work is partially supported by the Grant-in-Aid for Scientific Research (C) (No. 24540199) from Japan Society for the Promotion of Science. 


$$
\begin{aligned}
A(\mathrm{C}): & u^{(i)}(0)=u^{(i)}(1)=0 \quad(0 \leq i \leq M-1) \\
A(\mathrm{D}): & u^{(2 i)}(0)=u^{(2 i)}(1)=0 \quad(0 \leq i \leq[(M-1) / 2]), \\
A(\mathrm{~N}): & : u^{(2 i+1)}(0)=u^{(2 i+1)}(1)=0(0 \leq i \leq[(M-2) / 2]), \quad \int_{0}^{1} u(x) d x=0, \\
A(\mathrm{DN}): & u^{(2 i)}(0)=0 \quad(0 \leq i \leq[(M-1) / 2]), \\
& u^{(2 i+1)}(1)=0 \quad(0 \leq i \leq[(M-2) / 2]), \\
A(\mathrm{CF}): & u^{(i)}(0)=0 \quad(0 \leq i \leq M-1) .
\end{aligned}
$$

It should be noted that if $M=1$ the boundary conditions for $u$ in $A(\mathrm{~N})$ and for $u$ on $x=1$ in $A(\mathrm{DN})$ are not required. The script $\mathrm{X}$ means that $\mathrm{P}$ is Periodic, $\mathrm{AP}$ is Anti Periodic, $\mathrm{C}$ is Clamped, D is Dirichlet, $\mathrm{N}$ is Neumann, DN is Dirichlet-Neumann and CF is Clamped-Free boundary conditions. Let us consider $L^{p}$ Sobolev inequality including $j$-th derivative, where $j$ satisfies $j=0,1,2, \ldots, M-1$. We call this inequality $j$-th $L^{p}$ Sobolev inequality for short:

$$
\sup _{0 \leq y \leq 1}\left|u^{(j)}(y)\right| \leq C\left\|u^{(M)}\right\|_{p},
$$

where $u$ is an element of Sobolev space $W(\mathrm{X}, M, p)$. In our previous work, the best constants of $j$-th $L^{p}$ Sobolev inequality (1.1) were obtained in various boundary conditions as the following table.

\begin{tabular}{c|c|c|c}
\hline $\mathrm{X}$ & $j=0$ and $p=2$ & $j=0$ and $1<p<\infty$ & $j=1,2,3, \ldots$ and $1<p<\infty$ \\
\hline $\mathrm{P}$ & {$[9]$} & {$[1]$} & this paper \\
$\mathrm{AP}$ & {$[9]$} & - & - \\
$\mathrm{C}$ & {$[7]$} & $M=1,2,3[6]$ & - \\
$\mathrm{D}$ & {$[9]$} & $M=2 m[2], M=1,3,5[3]$ & - \\
$\mathrm{N}$ & {$[9]$} & {$[4]$} & this paper $(j=2 l)$ \\
$\mathrm{DN}$ & {$[9]$} & {$[10]$} & {$[10]$} \\
$\mathrm{CF}$ & {$[5]$} & {$[8]$} & {$[8]$} \\
\hline
\end{tabular}

From this table, we see that the difficulty in obtaining the best constant seems to increase in the case of $p \neq 2$. Indeed, each result in the case of $p \neq 2$ was obtained through a different method. No unified approach (maximizing the diagonal value of reproducing kernels; see $[5,7,9])$ as in the case of $p=2$ seems to exist for the case of $p \neq 2$.

In this paper, we would like to treat the case of $W(\mathrm{P}, M, p)$ and $W(\mathrm{~N}, M, p)$ and enrich the above table. To state the conclusion, we introduce Bernoulli polynomials $b_{k}(x)$ defined by the following recurrence relation:

$$
\left\{\begin{array}{l}
b_{0}(x)=1, \\
b_{k}^{\prime}(x)=b_{k-1}(x), \quad \int_{0}^{1} b_{k}(x) d x=0 \quad(k=1,2,3, \ldots) .
\end{array}\right.
$$

Hence, we have

$$
b_{1}(x)=x-\frac{1}{2}, \quad b_{2}(x)=\frac{x^{2}}{2}-\frac{x}{2}+\frac{1}{12}, \quad b_{3}(x)=\frac{x^{3}}{6}-\frac{x^{2}}{4}+\frac{x}{12}, \ldots
$$


and the auxiliary function $b_{m}(\alpha ; x)=b_{m}(x)-b_{m}(\alpha)$. These polynomials play an important role in this paper. The main result is as follows:

TheOREM 1.1. Let $M, m=1,2,3, \ldots, j=0,1, \ldots, M-1$ and $l=0,1,2, \ldots$. For any function $u \in W(\mathrm{P}, M, p)$, there exists a positive constant $C$ which is independent of $u$ such that $j$-th $L^{p}$ Sobolev inequality (1.1) holds. Moreover, the best constant $C(\mathrm{P}, M, j)$ of (1.1) is given by

$$
\begin{array}{llll}
C(\mathrm{P}, 2 m-1,2 l)= & \left\|b_{2(m-1-l)+1}\right\|_{q} & (M=2 m-1, & j=2 l), \\
C(\mathrm{P}, 2 m-1,2 l+1)= & \left\|b_{2(m-1-l)}\left(\alpha_{m-1-l} ; \cdot\right)\right\|_{q} & (M=2 m-1, & j=2 l+1), \\
C(\mathrm{P}, 2 m, 2 l)= & \left\|b_{2(m-l)}\left(\alpha_{m-l} ; \cdot\right)\right\|_{q} & (M=2 m, & j=2 l), \\
C(\mathrm{P}, 2 m, 2 l+1)= & \left\|b_{2(m-1-l)+1}\right\|_{q} & (M=2 m, & j=2 l+1) .
\end{array}
$$

From Lemma 2.1, we see that $\alpha=\alpha_{k}$ is the unique solution to the equation

$$
\partial_{\alpha}\left\|b_{2 k}(\alpha ; \cdot)\right\|_{q}^{q}=0 \quad(0<\alpha<1 / 2, k=0,1,2, \ldots) .
$$

If we replace $C$ by $C(\mathrm{P}, M, j)$ as (1.3), then the equality holds for $u(x)=c U(\mathrm{P}, M, j ; x)$ where $c$ is an arbitrary constant. $U(\mathrm{P}, M, j ; x)(0<x<1)$ is given by

$$
\begin{aligned}
& U(\mathrm{P}, 2 m-1,2 l ; x) \\
& =\int_{0}^{1} \operatorname{sgn}(x-y) b_{2 m-1}(|x-y|) \operatorname{sgn}\left(b_{2(m-1-l)+1}(y)\right)\left|b_{2(m-1-l)+1}(y)\right|^{q-1} d y, \\
& U(\mathrm{P}, 2 m-1,2 l+1 ; x) \\
& =\int_{0}^{1} \operatorname{sgn}(x-y) b_{2 m-1}(|x-y|) \operatorname{sgn}\left(b_{2(m-1-l)}\left(\alpha_{m-1-l} ; y\right)\right)\left|b_{2(m-1-l)}\left(\alpha_{m-1-l} ; y\right)\right|^{q-1} d y,
\end{aligned}
$$

$U(\mathrm{P}, 2 m, 2 l ; x)$

$=\int_{0}^{1} b_{2 m}(|x-y|) \operatorname{sgn}\left(b_{2(m-l)}\left(\alpha_{m-l} ; y\right)\right)\left|b_{2(m-l)}\left(\alpha_{m-l} ; y\right)\right|^{q-1} d y$,

$U(\mathrm{P}, 2 m, 2 l+1 ; x)$

$$
=\int_{0}^{1} b_{2 m}(|x-y|) \operatorname{sgn}\left(b_{2(m-1-l)+1}(y)\right)\left|b_{2(m-1-l)+1}(y)\right|^{q-1} d y \text {. }
$$

THEOREM 1.2. Let $M, m=1,2,3, \ldots, j=0,1, \ldots, M-1$ and $l=0,1,2, \ldots$ For any function $u \in W(\mathrm{~N}, M, p)$, there exists a positive constant $C$ which is independent of 
$u$ such that $j$-th $L^{p}$ Sobolev inequality (1.1) holds. Moreover, the best constant $C(\mathrm{~N}, M, j)$ of (1.1) is given by

$$
\begin{array}{llll}
C(\mathrm{~N}, 2 m-1,2 l)= & 2^{2(m-1-l)+1}\left\|b_{2(m-1-l)+1}\right\|_{q} & (M=2 m-1, & j=2 l), \\
C(\mathrm{~N}, 2 m, 2 l)= & 2^{2(m-l)}\left\|b_{2(m-l)}\left(\alpha_{m-l} ; \cdot\right)\right\|_{q} & (M=2 m, & j=2 l),
\end{array}
$$

where $\alpha_{m-l}$ is the unique solution to the equation (1.4) for $k=m-l$. If we replace $C$ by $C(\mathrm{~N}, M, j)$ as (1.9), then the equality holds for $u(x)=c V(\mathrm{P} 2, M, j ; x)$ where $c$ is an arbitrary constant. $V(\mathrm{P} 2, M, j ; x)(0<x<1)$ is given by

$$
\begin{aligned}
& V(\mathrm{P} 2,2 m-1,2 l ; x) \\
& =\int_{0}^{2} \operatorname{sgn}(x-y) b_{2 m-1}\left(\frac{|x-y|}{2}\right) \operatorname{sgn}\left(b_{2(m-1-l)+1}\left(\frac{y}{2}\right)\right)\left|b_{2(m-1-l)+1}\left(\frac{y}{2}\right)\right|^{q-1} d y,
\end{aligned}
$$

$V(\mathrm{P} 2,2 m, 2 l ; x)$

$$
=\int_{0}^{2} b_{2 m}\left(\frac{|x-y|}{2}\right) \operatorname{sgn}\left(b_{2(m-l)}\left(\alpha_{m-l} ; \frac{y}{2}\right)\right)\left|b_{2(m-l)}\left(\alpha_{m-l} ; \frac{y}{2}\right)\right|^{q-1} d y .
$$

REMARK 1. We could not obtain the result for the case of $M=2 m-1, j=2 l+1$ and $M=2 m, j=2 l+1$ for technical reason. The difficulties for these cases are briefly stated at the end of section 3 .

\section{Basic properties of Bernoulli polynomials}

In this section, we present the basic properties of Bernoulli polynomials. Bernoulli polynomials are introduced by the recurrence relation (1.2). Also, Bernoulli polynomials are defined by the generating function

$$
\frac{e^{x t}}{t^{-1}\left(e^{t}-1\right)}=\sum_{k=0}^{\infty} b_{k}(x) t^{k} \quad(|t|<2 \pi) .
$$

Bernoulli polynomial $b_{k}(x)$ is $k$-th polynomial with respect to $x$. Replacing $x$ to $1-x$ in (2.1), we have

$$
b_{k}(1-x)=(-1)^{k} b_{k}(x) \quad(k=0,1,2, \ldots) .
$$

Inserting $x=0$ and $x=1$ into (2.1), we have

$$
b_{k}(1)-b_{k}(0)= \begin{cases}1 & (k=1), \\ 0 & (k \neq 1) .\end{cases}
$$


From (2.2) and (2.3), we have

$$
b_{2 k+1}(0)= \begin{cases}-1 / 2 & (k=0), \\ 0 & (k=1,2,3, \ldots), \quad b_{2 k+1}(1 / 2)=0 \quad(k=0,1,2, \ldots) .\end{cases}
$$

Let $\{x\}$ be a decimal part of a real number $x$ as

$$
\{x\}=x-[x], \quad[x]=\sup \{n \in \mathbf{Z} \mid n \leq x\} .
$$

Since $\{x\}$ is a periodic function of $x$ with period 1, we derive Fourier expansion formula of $b_{k}(\{x\})$ as

$$
b_{k}(\{x\})=-\sum_{l \neq 0}(\sqrt{-1} 2 \pi l)^{-k} \exp (\sqrt{-1} 2 \pi l x) \quad(k=1,2,3, \ldots) .
$$

Hence we have

$$
b_{2 k}(\{x\})=(-1)^{k+1} 2 \sum_{l=1}^{\infty}(2 \pi l)^{-2 k} \cos (2 \pi l x) .
$$

Inserting $x=0$ and $x=1 / 2$ into the above relation, we have

$$
\begin{aligned}
& (-1)^{k+1} b_{2 k}(0)=\frac{2}{(2 \pi)^{2 k}} \zeta(2 k), \\
& (-1)^{k+1} b_{2 k}(1 / 2)=-\left(1-2^{-(2 k-1)}\right) \frac{2}{(2 \pi)^{2 k}} \zeta(2 k),
\end{aligned}
$$

where $\zeta(z)=\sum_{k=1}^{\infty} k^{-z}(\operatorname{Re} z>1)$ is Riemann-zeta function. So we have $\left|b_{2 k}(0)\right|>$ $\left|b_{2 k}(1 / 2)\right|$. For $k=2,3,4, \ldots$, the boundary value problem

$$
\left\{\begin{array}{l}
-\left(\frac{d}{d x}\right)^{2}\left((-1)^{k+1} b_{2 k-1}(x)\right)=(-1)^{k} b_{2 k-3}(x) \quad(0<x<1 / 2) \\
b_{2 k-1}(0)=b_{2 k-1}(1 / 2)=0
\end{array}\right.
$$

has a unique solution

$$
(-1)^{k+1} b_{2 k-1}(x)=\int_{0}^{1 / 2} G(x, y)(-1)^{k} b_{2 k-3}(y) d y,
$$

where $G(x, y)$ is positive-valued Green function given by

$$
G(x, y)=x \wedge y-2 x y=\min \{x, y\}-2 x y>0 \quad(0<x, y<1 / 2) .
$$

Starting from

$$
b_{1}(x)=x-\frac{1}{2}<0 \quad(0<x<1 / 2)
$$

we can show the following inequalities recurrently

$$
(-1)^{k+1} b_{2 k-1}(x)<0 \quad(0<x<1 / 2) .
$$


On the other hand, for any fixed $0 \leq \alpha \leq 1 / 2$, we define $b_{2 k}(\alpha ; x)$ as

$$
b_{2 k}(\alpha ; x)=b_{2 k}(x)-b_{2 k}(\alpha) \quad(k=1,2,3, \ldots, 0<x<1) .
$$

From (2.5), (2.6) and (2.7), we have

$$
\begin{aligned}
& (-1)^{k+1} b_{2 k}(\alpha ; x)=(-1)^{k+1}\left(b_{2 k}(x)-b_{2 k}(\alpha)\right) \begin{cases}>0 & (0 \leq x<\alpha), \\
=0 & (x=\alpha), \\
<0 & (\alpha<x \leq 1 / 2),\end{cases} \\
& \partial_{x}\left((-1)^{k+1} b_{2 k}(\alpha ; x)\right)=(-1)^{k+1} b_{2 k-1}(x)<0 \quad(0<x<1 / 2), \\
& \partial_{\alpha}\left((-1)^{k+1} b_{2 k}(\alpha ; x)\right)=(-1)^{k} b_{2 k-1}(\alpha)>0 \quad(0<\alpha<1 / 2) .
\end{aligned}
$$

LEMMA 2.1. The equation (1.4) with respect to $\alpha$ has a unique solution $\alpha=\alpha_{k}$.

Proof. We define the function $g(\alpha)$ as

$$
\begin{aligned}
g(\alpha) & =\left\|b_{2 k}(\alpha ; \cdot)\right\|_{q}^{q}=2 \int_{0}^{1 / 2}\left|b_{2 k}(\alpha ; x)\right|^{q} d x \\
& =2 \int_{0}^{\alpha}\left((-1)^{k+1} b_{2 k}(\alpha ; x)\right)^{q} d x+2 \int_{\alpha}^{1 / 2}\left((-1)^{k} b_{2 k}(\alpha ; x)\right)^{q} d x,
\end{aligned}
$$

where we use (2.2) and (2.8). Differentiating $g(\alpha)$ and using (2.10), we have

$$
g^{\prime}(\alpha)=\partial_{\alpha}\left\|b_{2 k}(\alpha ; \cdot)\right\|_{q}^{q}=2 q(-1)^{k} b_{2 k-1}(\alpha) h(\alpha),
$$

where

$$
h(\alpha)=\int_{0}^{\alpha}\left((-1)^{k+1} b_{2 k}(\alpha ; x)\right)^{q-1} d x-\int_{\alpha}^{1 / 2}\left((-1)^{k} b_{2 k}(\alpha ; x)\right)^{q-1} d x .
$$

From $q>1$ and (2.10), we have

$$
\begin{aligned}
h^{\prime}(\alpha)= & (q-1)(-1)^{k} b_{2 k-1}(\alpha) \\
& \times\left[\int_{0}^{\alpha}\left((-1)^{k+1} b_{2 k}(\alpha ; x)\right)^{q-2} d x+\int_{\alpha}^{1 / 2}\left((-1)^{k} b_{2 k}(\alpha ; x)\right)^{q-2} d x\right]>0
\end{aligned}
$$

for $0<\alpha<1 / 2$. Since $h(0)<0$ and $h(1 / 2)>0$, there exists a unique $\alpha_{k} \in(0,1 / 2)$ such that

$$
h(\alpha) \begin{cases}<0 & \left(0 \leq \alpha<\alpha_{k}\right) \\ =0 & \left(\alpha=\alpha_{k}\right) \\ >0 & \left(\alpha_{k}<\alpha \leq 1 / 2\right)\end{cases}
$$

So we have

$$
g^{\prime}(\alpha)=2 q(-1)^{k} b_{2 k-1}(\alpha) h(\alpha) \begin{cases}<0 & \left(0 \leq \alpha<\alpha_{k}\right), \\ =0 & \left(\alpha=\alpha_{k}\right), \\ >0 & \left(\alpha_{k}<\alpha \leq 1 / 2\right), \quad \min _{0 \leq \alpha \leq 1 / 2} g(\alpha)=g\left(\alpha_{k}\right) .\end{cases}
$$

This completes the proof of Lemma 2.1 . 
LEMMA 2.2. For any fixed $y(0 \leq y \leq 1)$, we have the following relations:

(1) $\left\|b_{k}(|\cdot-y|)\right\|_{q}^{q}=\left\|b_{k}\right\|_{q}^{q}$.

(2) $\left\|b_{k}(\alpha ;|\cdot-y|)\right\|_{q}^{q}=\left\|b_{k}(\alpha ; \cdot)\right\|_{q}^{q}$.

PROOF. (1) follows from

$$
\begin{aligned}
& \left\|b_{k}(|\cdot-y|)\right\|_{q}^{q}=\int_{0}^{1}\left|b_{k}(|x-y|)\right|^{q} d x=\int_{0}^{y}\left|b_{k}(y-x)\right|^{q} d x+\int_{y}^{1}\left|b_{k}(x-y)\right|^{q} d x \\
& \quad=\int_{0}^{y}\left|b_{k}(\xi)\right|^{q} d \xi+\int_{y}^{1}\left|b_{k}(1-\xi)\right|^{q} d \xi=\int_{0}^{y}\left|b_{k}(\xi)\right|^{q} d \xi+\int_{y}^{1}\left|(-1)^{k} b_{k}(\xi)\right|^{q} d \xi \\
& \quad=\int_{0}^{y}\left|b_{k}(\xi)\right|^{q} d \xi+\int_{y}^{1}\left|b_{k}(\xi)\right|^{q} d \xi=\int_{0}^{1}\left|b_{k}(\xi)\right|^{q} d \xi=\left\|b_{k}\right\|_{q}^{q},
\end{aligned}
$$

where we use (2.2). (2) is shown by the same way.

LEMMA 2.3.

$$
\begin{aligned}
& \text { (1) } \int_{0}^{1} \operatorname{sgn}\left(b_{2 k+1}(x)\right)\left|b_{2 k+1}(x)\right|^{q-1} d x=0 . \\
& \text { (2) } \int_{0}^{1} \operatorname{sgn}\left(b_{2 k}\left(\alpha_{k} ; x\right)\right)\left|b_{2 k}\left(\alpha_{k} ; x\right)\right|^{q-1} d x=0 .
\end{aligned}
$$

PROOF. Using (2.2), we have

$$
\operatorname{sgn}\left(b_{2 k+1}(1-x)\right)\left|b_{2 k+1}(1-x)\right|^{q-1}=-\operatorname{sgn}\left(b_{2 k+1}(x)\right)\left|b_{2 k+1}(x)\right|^{q-1} .
$$

So we have (1). Using Lemma 2.1, we have (2) since

$$
\begin{aligned}
& \int_{0}^{1} \operatorname{sgn}\left(b_{2 k}\left(\alpha_{k} ; x\right)\right)\left|b_{2 k}\left(\alpha_{k} ; x\right)\right|^{q-1} d x=2 \int_{0}^{1 / 2} \operatorname{sgn}\left(b_{2 k}\left(\alpha_{k} ; x\right)\right)\left|b_{2 k}\left(\alpha_{k} ; x\right)\right|^{q-1} d x \\
& =2\left[\int_{0}^{\alpha_{k}}\left((-1)^{k+1} b_{2 k}\left(\alpha_{k} ; x\right)\right)^{q-1} d x-\int_{\alpha_{k}}^{1 / 2}\left((-1)^{k} b_{2 k}\left(\alpha_{k} ; x\right)\right)^{q-1} d x\right]=2 h\left(\alpha_{k}\right)=0,
\end{aligned}
$$

where $h(\alpha)$ is defined by (2.11).

LEMMA 2.4. For $f(x)=\left|b_{k}(x)\right|^{q-1}$ and $g(x)=\left|b_{k}(\alpha ; x)\right|^{q-1}$, we have

$$
\|f\|_{p}=\left\|b_{k}\right\|_{q}^{q-1} \text { and }\|g\|_{p}=\left\|b_{k}(\alpha ; \cdot)\right\|_{q}^{q-1} \text {. }
$$

PROOF. Noting the relation $(q-1) p=q\left(1-\frac{1}{q}\right) p=q$, we have

$$
\begin{aligned}
\|f\|_{p} & =\left(\int_{0}^{1}|f(x)|^{p} d x\right)^{1 / p}=\left(\int_{0}^{1}\left|b_{k}(x)\right|^{(q-1) p} d x\right)^{1 / p}=\left(\int_{0}^{1}\left|b_{k}(x)\right|^{q} d x\right)^{1 / p} \\
& =\left\|b_{k}\right\|_{q}^{q / p}=\left\|b_{k}\right\|_{q}^{q-1} .
\end{aligned}
$$

The second relation is shown by the same way. 
Proposition 2.1. Let $N$ be $N=1,2,3, \ldots$ For any bounded continuous function $f(x)$ satisfying $\int_{0}^{1} f(y) d y=0$, the periodic boundary value problem

$$
\begin{aligned}
& \operatorname{BVP}(\mathrm{P}, N) \\
& \begin{cases}(-1)^{[(N+1) / 2]} u^{(N)}=f(x) & (0<x<1) \\
u^{(i)}(1)-u^{(i)}(0)=0 & (0 \leq i \leq N-1), \\
\int_{0}^{1} u(x) d x=0 & \end{cases}
\end{aligned}
$$

has a unique classical solution $u(x)$ expressed as

$$
u(x)=\int_{0}^{1} G(N ; x, y) f(y) d y \quad(0<x<1),
$$

where Green function $G(N ; x, y)$ is given by

$$
G(N ; x, y)=(-1)^{[(N+3) / 2]}(\operatorname{sgn}(x-y))^{N} b_{N}(|x-y|) \quad(0<x, y<1) .
$$

Especially, for $M=1,2,3, \ldots$, we have

$$
\begin{aligned}
& G(2 M-1 ; x, y)=(-1)^{M+1} \operatorname{sgn}(x-y) b_{2 M-1}(|x-y|), \\
& G(2 M ; x, y)=(-1)^{M+1} b_{2 M}(|x-y|) .
\end{aligned}
$$

Proof. See; Kametaka et al. [1]. Here, as an example, we would like to show the case $N=3$. Assume $N=3$, then using (2.2) (2.4), we have

$$
\begin{aligned}
& \int_{0}^{1} G(3 ; x, y) f(y) d y=\int_{0}^{1} G(3 ; x, y)(-1)^{2} u^{(3)}(y) d y \\
& =-\int_{0}^{x} b_{3}(x-y) u^{(3)}(y) d y+\int_{x}^{1} b_{3}(y-x) u^{(3)}(y) d y \\
& =-\left[b_{3}(x-y) u^{(2)}(y)+b_{2}(x-y) u^{(1)}(y)+b_{1}(x-y) u(y)\right]_{y=0}^{y=x} \\
& \quad+\left[b_{3}(y-x) u^{(2)}(y)-b_{2}(y-x) u^{(1)}(y)+b_{1}(y-x) u(y)\right]_{y=x}^{y=1}-\int_{0}^{x} u(y) d y-\int_{x}^{1} u(y) d y \\
& =u(x)-b_{3}(x)\left(u^{(2)}(1)-u^{(2)}(0)\right)-b_{2}(x)\left(u^{(1)}(1)-u^{(1)}(0)\right)-b_{1}(x)(u(1)-u(0)) \\
& \quad-\int_{0}^{1} u(y) d y .
\end{aligned}
$$

Using the boundary condition and orthogonality condition of $\mathrm{BVP}(\mathrm{P}, 3)$, we have (2.12) and (2.13) in the case of $N=3$.

LEMMA 2.5. The following relations hold if $0<x<1,0<y<1$ and $x \neq y$.

$$
\partial_{y}^{k} \partial_{x}^{M} G(2 M ; x, y)=(-1)^{M+1+k}(\operatorname{sgn}(x-y))^{M+k} b_{M-k}(|x-y|)
$$




$$
=\left\{\begin{array}{lll}
\operatorname{sgn}(x-y) b_{2(m-1-l)+1}(|x-y|) & (M=2 m-1, & k=2 l), \\
-b_{2(m-1-l)}(|x-y|) & (M=2 m-1, & k=2 l+1), \\
-b_{2(m-l)}(|x-y|) & (M=2 m, & k=2 l), \\
\operatorname{sgn}(x-y) b_{2(m-1-l)+1}(|x-y|) & (M=2 m, & k=2 l+1) .
\end{array}\right.
$$

PROOF. Noting the relations

$$
\partial_{x}|x-y|=\operatorname{sgn}(x-y), \quad \partial_{y}|x-y|=-\operatorname{sgn}(x-y),
$$

we have this lemma.

LEMMA 2.6. For any $u \in W(\mathrm{P}, M, p)$ and for any fixed $y(0 \leq y \leq 1)$, the following reproducing relation holds:

$$
u^{(j)}(y)=\int_{0}^{1} u^{(M)}(x) \partial_{y}^{j} \partial_{x}^{M} G(2 M ; x, y) d x .
$$

ProOF. Using Lemma 2.5, we have

$$
\begin{aligned}
I & =\int_{0}^{1} u^{(M)}(x) \partial_{y}^{j} \partial_{x}^{M} G(2 M ; x, y) d x \\
& =\int_{0}^{1} u^{(M)}(x)(-1)^{M+1+j}(\operatorname{sgn}(x-y))^{M+j} b_{M-j}(|x-y|) d x \\
& =-\int_{0}^{y} u^{(M)}(x) b_{M-j}(y-x) d x-(-1)^{M+j} \int_{y}^{1} u^{(M)}(x) b_{M-j}(x-y) d x \\
& =-I_{1}-I_{2}
\end{aligned}
$$

where

$$
\begin{aligned}
I_{1} & =\int_{0}^{y} u^{(M)}(x) b_{M-j}(y-x) d x \\
& =\sum_{k=0}^{M-1-j}\left[u^{(M-1-k)}(y) b_{M-j-k}(0)-u^{(M-1-k)}(0) b_{M-j-k}(y)\right]+\int_{0}^{y} u^{(j)}(x) d x
\end{aligned}
$$

and

$$
\begin{aligned}
I_{2}= & (-1)^{M+j} \int_{y}^{1} u^{(M)}(x) b_{M-j}(x-y) d x \\
= & \sum_{k=0}^{M-1-j}(-1)^{M+j+k}\left[u^{(M-1-k)}(1) b_{M-j-k}(1-y)-u^{(M-1-k)}(y) b_{M-j-k}(0)\right] \\
& +\int_{y}^{1} u^{(j)}(x) d x .
\end{aligned}
$$


So we have

$$
\begin{aligned}
& I=-I_{1}-I_{2} \\
& =\sum_{k=0}^{M-1-j}\left[-u^{(M-1-k)}(y) b_{M-j-k}(0)+u^{(M-1-k)}(0) b_{M-j-k}(y)\right. \\
& \left.\quad-u^{(M-1-k)}(1)(-1)^{M+j+k} b_{M-j-k}(1-y)+u^{(M-1-k)}(y)(-1)^{M+j+k} b_{M-j-k}(0)\right] \\
& \quad-\int_{0}^{1} u^{(j)}(x) d x .
\end{aligned}
$$

Using (2.2), we have

$$
\begin{aligned}
I= & \sum_{k=0}^{M-1-j}\left[\left(-1+(-1)^{M+j+k}\right) b_{M-j-k}(0) u^{(M-1-k)}(y)\right. \\
& \left.-\left(u^{(M-1-k)}(1)-u^{(M-1-k)}(0)\right) b_{M-j-k}(y)\right]-\int_{0}^{1} u^{(j)}(x) d x .
\end{aligned}
$$

For

$$
\begin{aligned}
(-1 & \left.+(-1)^{M+j+k}\right) b_{M-j-k}(0) u^{(M-1-k)}(y) \\
& =\left(-1+(-1)^{M-j-k}\right) b_{M-j-k}(0) u^{(M-1-k)}(y),
\end{aligned}
$$

setting $l=M-j-k(1 \leq l \leq M-j)$ and using (2.4), we have

$$
\left(-1+(-1)^{l}\right) b_{l}(0) u^{(l+j-1)}(y)= \begin{cases}u^{(j)}(y) & (l=1), \\ 0 & (2 \leq l \leq M-j) .\end{cases}
$$

So we have

$$
\begin{aligned}
& I=u^{(j)}(y)-\sum_{k=0}^{M-1-j}\left(u^{(M-1-k)}(1)-u^{(M-1-k)}(0)\right) b_{M-j-k}(y)-\int_{0}^{1} u^{(j)}(x) d x \\
& =\left\{\begin{array}{l}
u(y)-\sum_{k=0}^{M-1}\left(u^{(M-1-k)}(1)-u^{(M-1-k)}(0)\right) b_{M-k}(y)-\int_{0}^{1} u(x) d x \\
(j=0), \\
u^{(j)}(y)-\sum_{k=0}^{M-j}\left(u^{(M-1-k)}(1)-u^{(M-1-k)}(0)\right) b_{M-j-k}(y) \\
(j=1,2,3, \ldots, M-1) .
\end{array}\right.
\end{aligned}
$$

If we chose $u \in W(\mathrm{P}, M, p)$, then we have Lemma 2.6. 


\section{Proof of Theorems}

3.1. Proof of Theorem 1.1. We put the number as

$$
\begin{array}{lll}
\text { (I) } & M=2 m-1, & j=2 l, \\
\text { (II) } & M=2 m-1, & j=2 l+1, \\
\text { (III) } & M=2 m, & j=2 l, \\
\text { (IV) } & M=2 m, & j=2 l+1 .
\end{array}
$$

For any $u \in W(\mathrm{P}, M, p)$ and any fixed $y(0 \leq y \leq 1)$, using Lemma 2.5, the reproducing relation Lemma 2.6 is given by

$$
u^{(j)}(y)=\int_{0}^{1} u^{(M)}(x)\left\{\begin{array}{ll}
\operatorname{sgn}(x-y) b_{2(m-1-l)+1}(|x-y|) & \text { (I) } \\
-b_{2(m-1-l)}(|x-y|) & \text { (II) } \\
-b_{2(m-l)}(|x-y|) & \text { (III) } \\
\operatorname{sgn}(x-y) b_{2(m-1-l)+1}(|x-y|) & \text { (IV) }
\end{array}\right\} d x .
$$

For (II) and (III), we add the correction term $b_{2(m-1-l)}\left(\alpha_{m-1-l}\right)$ and $b_{2(m-l)}\left(\alpha_{m-l}\right)$, respectively. Noting

$$
\int_{0}^{1} u^{(M)}(x) d x=u^{(M-1)}(1)-u^{(M-1)}(0)=0,
$$

we have

$$
u^{(j)}(y)=\int_{0}^{1} u^{(M)}(x)\left\{\begin{array}{ll}
\operatorname{sgn}(x-y) b_{2(m-1-l)+1}(|x-y|) & \text { (I) } \\
-b_{2(m-1-l)}\left(\alpha_{m-1-l} ;|x-y|\right) & \text { (II) } \\
-b_{2(m-l)}\left(\alpha_{m-l} ;|x-y|\right) & \text { (III) } \\
\operatorname{sgn}(x-y) b_{2(m-1-l)+1}(|x-y|) & \text { (IV) }
\end{array}\right\} d x
$$

Applying Hölder inequality to the above relation, using Lemma 2.2 and taking the supremum of the both sides with respect to $y$, we have $j$-th $L^{p}$ Sobolev inequality:

$$
\sup _{0 \leq y \leq 1}\left|u^{(j)}(y)\right| \leq\left\{\begin{array}{ll}
\left\|b_{2(m-1-l)+1}\right\|_{q} & \text { (I) } \\
\left\|b_{2(m-1-l)}\left(\alpha_{m-1-l} ; \cdot\right)\right\|_{q} & \text { (II) } \\
\left\|b_{2(m-l)}\left(\alpha_{m-l} ; \cdot\right)\right\|_{q} & \text { (III) } \\
\left\|b_{2(m-1-l)+1}\right\|_{q} & \text { (IV) }
\end{array}\right\}\left\|u^{(M)}\right\|_{p}=C(M, j)\left\|u^{(M)}\right\|_{p} .
$$


The equality holds for $u(x)=U(x)=U(M, j ; x)$ which satisfy $U^{(M)}(M, j ; x)=$ $F(M, j ; x)$, where

$$
F(M, j ; x)=\left\{\begin{array}{ll}
\operatorname{sgn}\left(b_{2(m-1-l)+1}(x)\right)\left|b_{2(m-1-l)+1}(x)\right|^{q-1} & \text { (I) } \\
\operatorname{sgn}\left(b_{2(m-1-l)}\left(\alpha_{m-1-l} ; x\right)\right)\left|b_{2(m-1-l)}\left(\alpha_{m-1-l} ; x\right)\right|^{q-1} & \text { (II) } \\
\operatorname{sgn}\left(b_{2(m-l)}\left(\alpha_{m-l} ; x\right)\right)\left|b_{2(m-l)}\left(\alpha_{m-l} ; x\right)\right|^{q-1} & \text { (III) } \\
\operatorname{sgn}\left(b_{2(m-1-l)+1}(x)\right)\left|b_{2(m-1-l)+1}(x)\right|^{q-1} & \text { (IV) }
\end{array}\right\}
$$

On the other hand, from Lemma 2.3, we see that

$$
\int_{0}^{1} F(M, j ; x) d x=0 .
$$

Thus $U(M, j ; x)$ given by $(1.5) \sim(1.8)$ satisfies $\mathrm{BVP}(\mathrm{P}, M)$, so $U(M, j ; x) \in W(\mathrm{P}, M, p)$ and $U(M, j ; x)$ achieves the equality in (3.1). In fact, using Lemma 2.4, we have

$$
\left|U^{(j)}(0)\right|=\left\{\begin{array}{ll}
\left\|b_{2(m-1-l)+1}\right\|_{q}^{q} & \text { (I) } \\
\left\|b_{2(m-1-l)}\left(\alpha_{m-1-l} ; \cdot\right)\right\|_{q}^{q} & (\mathrm{II}) \\
\left\|b_{2(m-l)}\left(\alpha_{m-l} ; \cdot\right)\right\|_{q}^{q} & (\mathrm{III}) \\
\left\|b_{2(m-1-l)+1}\right\|_{q}^{q} & (\mathrm{IV})
\end{array}\right\}=C(M, j)\|F\|_{p}=C(M, j)\left\|U^{(M)}\right\|_{p} .
$$

Combining this with (3.1), we have

$$
C(M, j)\left\|U^{(M)}\right\|_{p}=\left|U^{(j)}(0)\right| \leq \sup _{0 \leq y \leq 1}\left|U^{(j)}(y)\right| \leq C(M, j)\left\|U^{(M)}\right\|_{p} .
$$

This shows that $C(M, j)$ is the best constant of $j$-th $L^{p}$ Sobolev inequality (3.1) and the equality holds for $U(M, j ; x)$ defined by $(1.5) \sim(1.8)$. This completes the proof of Theorem 1.1 .

3.2. Proof of Theorem 1.2. To prove Theorem 1.2, we prepare the following lemma.

LEMMA 3.1. $V(\mathrm{P} 2, M, 2 l ; x)$ defined by (1.10) and (1.11) in Theorem 1.2 satisfies the following properties.

$$
\begin{aligned}
& V(\mathrm{P} 2, M, 2 l ; 2-x)=V(\mathrm{P} 2, M, 2 l ; x) \quad(0<x<1) . \\
& V^{(2 i+1)}(\mathrm{P} 2, M, 2 l ; 0)=0 \quad\left(0 \leq i \leq\left[\frac{M-2}{2}\right]\right) . \\
& V^{(2 i+1)}(\mathrm{P} 2, M, 2 l ; 1)=0 \quad\left(0 \leq i \leq\left[\frac{M-2}{2}\right]\right) . \\
& V^{(i)}(\mathrm{P} 2, M, 2 l ; 2)-V^{(i)}(\mathrm{P} 2, M, 2 l ; 0)=0 \quad(0 \leq i \leq M-1) . \\
& \int_{0}^{2} V(\mathrm{P} 2, M, 2 l ; x) d x=0 .
\end{aligned}
$$




$$
\int_{0}^{1} V(\mathrm{P} 2, M, 2 l ; x) d x=0 .
$$

Proof. We only prove the case $M=2 m-1$. From (2.2), we have (3.2) since $V(\mathrm{P} 2,2 m-1,2 l ; 2-x)$

$$
\begin{aligned}
& =\int_{0}^{2} \operatorname{sgn}(2-x-y) b_{2 m-1}\left(\frac{|2-x-y|}{2}\right) \operatorname{sgn}\left(b_{2(m-1-l)+1}\left(\frac{y}{2}\right)\right)\left|b_{2(m-1-l)+1}\left(\frac{y}{2}\right)\right|^{q-1} d y \\
& =\int_{0}^{2} \operatorname{sgn}(z-x) b_{2 m-1}\left(\frac{|z-x|}{2}\right) \operatorname{sgn}\left(b_{2(m-1-l)+1}\left(\frac{2-z}{2}\right)\right)\left|b_{2(m-1-l)+1}\left(\frac{2-z}{2}\right)\right|^{q-1} d z \\
& =\int_{0}^{2} \operatorname{sgn}(x-z) b_{2 m-1}\left(\frac{|x-z|}{2}\right) \operatorname{sgn}\left(b_{2(m-1-l)+1}\left(\frac{z}{2}\right)\right)\left|b_{2(m-1-l)+1}\left(\frac{z}{2}\right)\right|^{q-1} d z \\
& =V(\mathrm{P} 2,2 m-1,2 l ; x) .
\end{aligned}
$$

Again by (2.2), we obtain (3.3) since

$$
\begin{aligned}
& V^{(2 i+1)}(\mathrm{P} 2,2 m-1,2 l ; 0) \\
&= \int_{0}^{2} 2^{-(2 i+1)} b_{2(m-1-i)}\left(\frac{y}{2}\right) \operatorname{sgn}\left(b_{2(m-1-l)+1}\left(\frac{y}{2}\right)\right)\left|b_{2(m-1-l)+1}\left(\frac{y}{2}\right)\right|^{q-1} d y \\
&=-\int_{0}^{2} 2^{-(2 i+1)} b_{2(m-1-i)}\left(\frac{2-y}{2}\right) \operatorname{sgn}\left(b_{2(m-1-l)+1}\left(\frac{2-y}{2}\right)\right) \\
& \times\left|b_{2(m-1-l)+1}\left(\frac{2-y}{2}\right)\right|^{q-1} d y \\
&=-\int_{0}^{2} 2^{-(2 i+1)} b_{2(m-1-i)}\left(\frac{z}{2}\right) \operatorname{sgn}\left(b_{2(m-1-l)+1}\left(\frac{z}{2}\right)\right)\left|b_{2(m-1-l)+1}\left(\frac{z}{2}\right)\right|^{q-1} d y \\
&=-V^{(2 i+1)}(\mathrm{P} 2,2 m-1,2 l ; 0) .
\end{aligned}
$$

Differentiating (3.2), $2 i$ and $2 i+1$ times, we have

$$
\begin{aligned}
& V^{(2 i)}(\mathrm{P} 2,2 m-1,2 l ; 2-x)=V^{(2 i)}(\mathrm{P} 2,2 m-1,2 l ; x), \\
& -V^{(2 i+1)}(\mathrm{P} 2,2 m-1,2 l ; 2-x)=V^{(2 i+1)}(\mathrm{P} 2,2 m-1,2 l ; x) .
\end{aligned}
$$

Putting $x=1$, we have

$$
-V^{(2 i+1)}(\mathrm{P} 2,2 m-1,2 l ; 1)=V^{(2 i+1)}(\mathrm{P} 2,2 m-1,2 l ; 1) .
$$

So we have (3.4). Putting $x=0$, we have

$$
\begin{aligned}
& V^{(2 i)}(\mathrm{P} 2,2 m-1,2 l ; 2)=V^{(2 i)}(\mathrm{P} 2,2 m-1,2 l ; 0), \\
& -V^{(2 i+1)}(\mathrm{P} 2,2 m-1,2 l ; 2)=V^{(2 i+1)}(\mathrm{P} 2,2 m-1,2 l ; 0)=0,
\end{aligned}
$$


where we use (3.3). So we have (3.5). From

$$
\begin{aligned}
& \int_{0}^{2} \operatorname{sgn}(x-y) b_{2 m-1}\left(\frac{|x-y|}{2}\right) d x=-\int_{0}^{y} b_{2 m-1}\left(\frac{y-x}{2}\right) d x+\int_{y}^{2} b_{2 m-1}\left(\frac{x-y}{2}\right) d x \\
& =-2 \int_{0}^{y / 2} b_{2 m-1}(z) d z+2 \int_{y / 2}^{1} b_{2 m-1}(1-z) d z=-2 \int_{0}^{1} b_{2 m-1}(z) d z=0,
\end{aligned}
$$

we have (3.6). From (3.2) and (3.6), we have (3.7).

Now we prove Theorem 1.2.

Proof of Theorem 1.2. We define the space $W(\mathrm{P} 2, M, p)$ as

$$
W(\mathrm{P} 2, M, p)=\left\{u \mid u^{(M)} \in L^{p}(0,2), u \text { satisfies } A(\mathrm{P} 2)\right\},
$$

where

$$
A(\mathrm{P} 2): \quad u^{(i)}(2)-u^{(i)}(0)=0 \quad(0 \leq i \leq M-1), \quad \int_{0}^{2} u(x) d x=0 .
$$

For any $u \in W(\mathrm{~N}, M, p)$, we define $\widetilde{u}(x)(0 \leq x \leq 2)$ as

$$
\tilde{u}(x)= \begin{cases}u(x) & (0 \leq x \leq 1), \\ u(2-x) & (1 \leq x \leq 2) .\end{cases}
$$

Since $u \in W(\mathrm{~N}, M, p)$ satisfies Neumann boundary condition at $x=0$ and $x=1$, it is easy to see that $\tilde{u}$ is an element of $W(\mathrm{P} 2, M, p)$. So, we have

$$
\begin{aligned}
& \sup _{0 \leq y \leq 1}\left|u^{(j)}(y)\right|=\sup _{0 \leq y \leq 2}\left|\tilde{u}^{(j)}(y)\right| \\
& \quad \leq C(\mathrm{P} 2, M, j)\left\|\widetilde{u}^{(M)}\right\|_{L^{p}(0,2)}=2^{1 / p} C(\mathrm{P} 2, M, j)\left\|u^{(M)}\right\|_{L^{p}(0,1)},
\end{aligned}
$$

where $C(\mathrm{P} 2, M, j)$ is the best constant of $j$-th $L^{p}$ Sobolev inequality:

$$
\sup _{0 \leq y \leq 2}\left|u^{(j)}(y)\right| \leq C\left\|u^{(M)}\right\|_{L^{p}(0,2)} \quad(\forall u \in W(\mathrm{P} 2, M, p)) .
$$

Note that by simple computation, it holds that $C(\mathrm{P} 2, M, j)=2^{M-j-1 / p} C(\mathrm{P}, M, j)$ (See; Appendix). Next, we construct the function which attains the equality in (3.8) when $j=$ 2l. Let $\tilde{u}_{0}(x)=V(\mathrm{P} 2, M, 2 l ; x)(0 \leq x \leq 2)$. From Lemma 3.1, $\tilde{u}_{0} \in W(\mathrm{P} 2, M, p)$. Substituting $\widetilde{u}_{0}$ into (3.8), from Theorem 1.1, we have the equality in (3.8). Let $\widetilde{\widetilde{u}}_{0}$ be the restriction of $\widetilde{u}_{0}$ on $[0,1]$. From Lemma 3.1, we see that $\widetilde{\widetilde{u}}_{0} \in W(\mathrm{~N}, M, p)$ and attains the equality of the following inequality

$$
\sup _{0 \leq y \leq 1}\left|u^{(2 l)}\right| \leq 2^{1 / p} C(\mathrm{P} 2, M, 2 l)\left\|u^{(M)}\right\|_{L^{p}(0,1)}=2^{M-2 l} C(\mathrm{P}, M, 2 l)\left\|u^{(M)}\right\|_{L^{p}(0,1)} .
$$

This proves Theorem 1.2. 


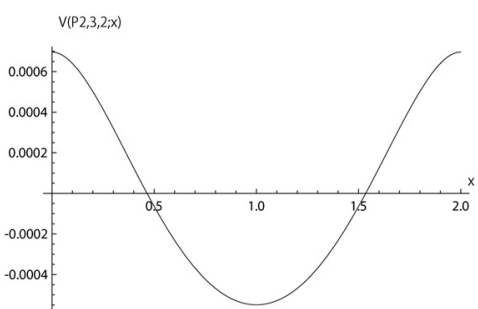

(i)

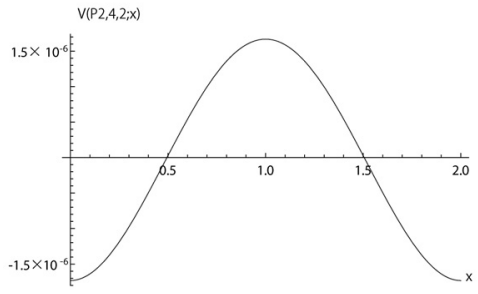

(iii)

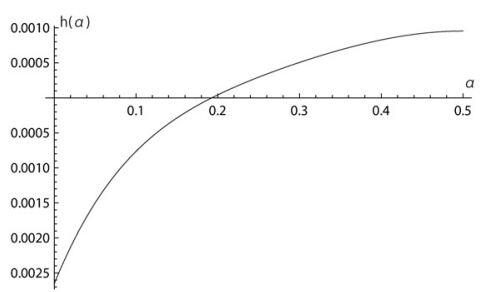

(ii)

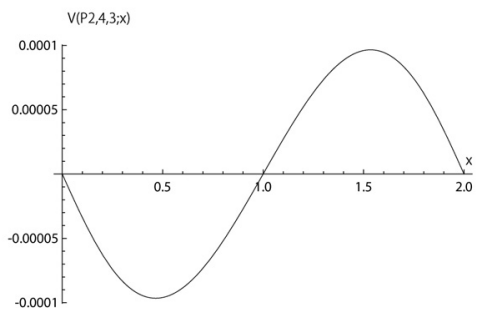

(iv)

FIgURE 1. The graphs of (i) $V(\mathrm{P} 2,3,2 ; x)$, (ii) $h(\alpha)$, (iii) $V(\mathrm{P} 2,4,2 ; x)$ for $\alpha_{1}=$ 0.193 and (iv) $V(\mathrm{P} 2,4,3 ; x)$ for $q=3.2$.

Figure 1 shows the graphs of $V(\mathrm{P} 2, M, j ; x)$. From Figure (i) $(M=3, j=2, q=3.2)$ and (iii) $(M=4, j=2, q=3.2)$, we see that Neumann boundary condition is satisfied at both $x=0$ and $x=1$. Figure (ii) shows the graph of $h(\alpha)$ for $M=4, k=m-l=2-1=1$. From (ii), we see the root of $h(\alpha)=0$ is $\alpha_{1}=0.193$. We used this value for drawing the figure (iii). Figure (iv) shows the graph of $V(\mathrm{P} 2,4,3 ; x)$ for $q=3.2$. From (iv), we see that Neumann boundary condition is not satisfied at both $x=0$ and $x=1$. Moreover, $x=0.5$ and 1.5 are not critical point of $V(\mathrm{P} 2,4,3 ; x)$. Thus we can not apply the proof of Theorem 1.2 for the case $M=2 m$ and $j=2 l+1$. The case $M=2 m-1$ and $j=2 l+1$ also remains for the same reason. Therefore we need yet another method for these two cases. But we have not any at this time.

\section{Appendix}

For

$$
\sup _{0 \leq y \leq 2}\left|u^{(j)}(y)\right| \leq C(\mathrm{P} 2, M, j)\left\|u^{(M)}\right\|_{L^{p}(0,2)} \quad(\forall u \in W(\mathrm{P} 2, M, p)),
$$

we put

$$
u(x)=\left.v(\xi)\right|_{\xi=x / 2} \quad(0<x<2,0<\xi<1)
$$


Differentiating this function $i$ times with respect to $x$, we have

$$
u^{(i)}(x)=2^{-i} v^{(i)}(\xi) \quad(i=0,1,2, \ldots, M) .
$$

Since $u \in W(\mathrm{P} 2, M, p)$, we have

$$
2^{-i} v^{(i)}(1)=u^{(i)}(2)=u^{(i)}(0)=2^{-i} v^{(i)}(0) \quad(0 \leq i \leq M-1),
$$

and

$$
0=\int_{0}^{2} u(x) d x=2 \int_{0}^{1} v(\xi) d \xi .
$$

So we have $v \in W(\mathrm{P}, M, p)$. Moreover, from

$$
\left\|u^{(M)}\right\|_{L^{p}(0,2)}^{p}=\int_{0}^{2}\left|u^{(M)}(x)\right|^{p} d x=\int_{0}^{1} 2^{-M p}\left|v^{(M)}(\xi)\right|^{p} 2 d \xi=2^{-M p+1}\left\|v^{(M)}\right\|_{L^{p}(0,1)}^{p},
$$

we have

$$
\left\|u^{(M)}\right\|_{L^{p}(0,2)}=2^{-M+1 / p}\left\|v^{(M)}\right\|_{L^{p}(0,1)} .
$$

Hence, for (3.9), we have

$$
\begin{aligned}
& \sup _{0 \leq \eta \leq 1}\left|2^{-j} v^{(j)}(\eta)\right|=\sup _{0 \leq y \leq 2}\left|u^{(j)}(y)\right| \\
& \leq C(\mathrm{P} 2, M, j)\left\|u^{(M)}\right\|_{L^{p}(0,2)}=C(\mathrm{P} 2, M, j) 2^{-M+1 / p}\left\|v^{(M)}\right\|_{L^{p}(0,1)} .
\end{aligned}
$$

So we have

$$
\begin{aligned}
& \sup _{0 \leq \eta \leq 1}\left|v^{(j)}(\eta)\right| \leq 2^{-M+j+1 / p} C(\mathrm{P} 2, M, j)\left\|v^{(M)}\right\|_{L^{p}(0,1)} \\
& (\forall v \in W(\mathrm{P}, M, p)) .
\end{aligned}
$$

Since there is an element which attains the equality above,

$$
2^{-M+j+1 / p} C(\mathrm{P} 2, M, j)=C(\mathrm{P}, M, j) .
$$

Thus we have

$$
C(\mathrm{P} 2, M, j)=2^{M-j-1 / p} C(\mathrm{P}, M, j) .
$$

\section{References}

[ 1 ] Y. Kametaka, Y. Oshime, K. Watanabe, H. Yamagishi, A. Nagai and K. Takemura, The best constant of $L^{p}$ Sobolev inequality corresponding to the periodic boundary value problem for $(-1)^{M}(d / d x)^{2 M}$, Sci. Math. Jpn. e-2007 (2007), 269-281.

[ 2 ] Y. Oshime, Y. KametaKa and H. Yamagishi, The best constant of $L^{p}$ Sobolev inequality corresponding to Dirichlet boundary value problem for $(d / d x)^{4 m}$, Sci. Math. Jpn. e-2008 (2008), 461-469.

[ 3 ] Y. Oshime and K. WATANABE, The best constant of $L^{p}$ Sobolev inequality corresponding to Dirichlet boundary value problem II, Tokyo J. Math. 34 (2011) 115-133. 
[ 4 ] Y. Oshime, H. Yamagishi and K. Watanabe, The best constant of $L^{p}$ Sobolev inequality corresponding to Neumann boundary value problem for $(-1)^{M}(d / d x)^{2 M}$, Hiroshima Math. J. 42 (2012), 293-299.

[ 5 ] K. TAKEMURA, The beat constant of Sobolev inequality corresponding to clamped-free boundary value problem for $(-1)^{M}(d / d x)^{2 M}$, Proc. Jpn. Acad. 85 (2009), 112-117.

[6] K. Watanabe, Y. Kametaka, A. Nagai, H. Yamagishi and K. Takemura, Symmetrization of functions and the best constant of 1-dim $L^{p}$ Sobolev inequality, J. Inequal. Appl. Vol. 2009, Article ID 874631.

[ 7 ] K. Watanabe, Y. Kametaka, H. Yamagishi, A. Nagai and K. Takemura, The best constant of Sobolev inequality corresponding to clamped boundary value problem, Bound. Value Probl. Vol. 2011, Article ID 875057.

[ 8 ] K. Watanabe, K. Takemura, Y. Kametaka, A. Nagai and H. Yamagishi, Lyapunov-type inequalities for $2 M$ th order equations under clamped-free boundary conditions, J. Inequal. Appl. Vol. 2012, Article ID 2012:242.

[ 9 ] H. Yamagishi, Y. Kametaka, A. Nagai, K. Watanabe and K. TAKemura, Riemann zeta function and the best constants of five series of Sobolev inequalities, RIMS Kokyuroku Bessatsu B13 (2009), 125-139.

[10] H. Yamagishi, K. WAtAnABE and Y. KametaKA, The best constant of $L^{p}$ Sobolev inequality corresponding to Dirichlet-Neumann boundary value problem, Math. J. Okayama Univ. 56 (2014), 145-155.

Present Addresses:

HIROYUKI YAMAGISHI

TOKYO METROPOLITAN COLLEGE OF INDUSTRIAL TECHNOLOGY, 1-10-40 Higashi-OI, ShinagaWa, TOKYo 140-0011, JAPAN.

e-mail: yamagisi@s.metro-cit.ac.jp

Kohtaro Watanabe

Department of Computer Science, National Defense Academy,

1-10-20 HASHIRIMIZU, YOKOSUKA 239-8686, JAPAN.

e-mail:wata@nda.ac.jp 